\title{
An update on penicillium marneffei infection and advances in laboratory diagnosis
}

\section{Introduction}

Penicillium marneffei is an emerging pathogenic fungus that can cause systemic mycosis among human immunodeficiency virus infected patients. The species Penicillium marneffei belong to genus Penicillium which has more than 250 known species, widely distributed in nature found in soil as well as decomposed organic debris. These fungi are rapid growers, conidial structure resembling brushes and produces musty odor. Human population may be adversely affected due to environmental contamination and these fungi traditionally have not been considered as very pathogenic and rarely are they implicated as etiological agent in a healthy human. However Penicillium marneffei causes infection in individuals with lower immunity. Penicillium marneffei is a unique dimorphic fungus and is responsible for causing clinical entity popularly called as Penicilliosis marneffei. ${ }^{1,2}$ This is an opportunistic fungal disease endemic in south East Asia. ${ }^{3}$ Pandemic of AIDS has virtually acted as trigger to rapid spread of penicilliosis marneffei as opportunistic infection or secondary infection. It is also known as AIDS defining illness among patients who have either lived or visited endemic areas regardless of time period since exposure. ${ }^{3}$

P. marneffei infection is endemic in various part of world including countries of south-east Asia and other region of tropical countries. ${ }^{4}$ The importance of $P$. marneffei most of time are related to HIV pandemic. Movement of people from one part to another part of world from endemic to non endemic increases its spread. ${ }^{5}$ It was found to be the third most frequent opportunistic pathogen after tuberculosis and cryptococcosis among immunosuppressed in endemic areas. ${ }^{1}$

Clinical picture: Low-grade fever, weight loss, and skin lesions are common. Other characteristics are malaise, anemia and leukocytosis. Fungemia, generalized lymphadenopathy, and cough are also reported in many of patients. Subcutaneous and mucosal lesions, diarrhea, colonic lesions, hepatomegaly with or without splenomegaly, hemoptysis, osteoarticular lesions, and pericarditis have also been described. Skin lesions commonly occur on the face, upper trunk, and extremities. They may occur as papules, pustules, nodules, ulcers, or abscesses. In HIV-infected individuals, lesions commonly become umbilicated and resemble those of molluscum contagiosum. Pharyngeal and palatal lesions are also more commonly seen in HIVinfected patients. Lung lesions can appear as reticulonodular, nodular, or diffuse alveolar infiltrates, but on occasion they are cavitary and cause hemoptysis. Autopsy studies have revealed involvement of lymph nodes, liver, spleen, lung, kidney, skin, bone, bone marrow, adrenal, tonsil, bowel, and meninges. Patients who do not receive the appropriate antifungal treatment have a poor prognosis; however, primary treatment with amphotericin B and secondary prophylaxis with Itraconazole are effective.

Laboratory diagnoses of $P$. marneffei infection need demonstration of intracellular $P$. marneffei yeast cells in the infected tissue. Cultivating the fungus from clinical specimens is another means. In microscopy $P$. marneffei appears as a unicellular organism with round to oval cells or cross wall formation within macrophages, or form extracellular elongated cells. ${ }^{6}$ The determination of the
Volume 2 Issue I - 2016

\section{Ravi kant, Satish Gupte, Tanveer Kaur, Mandeep Kaur}

Department of Microbiology, Gian Sagar Medical College \& Hospital, India

Correspondence: Satish Gupte, Department of Microbiology, Gian Sagar Medical College \& Hospital, Rajpura, India, Email drsatishgupte@hotmail.com

Received: March 01, 2016 | Published: April 13, 2016

P. marneffei may be carried out by molecular techniques and such analyses will helps to understand the molecular mechanism of fungal morphogenesis, pathogenesis and host-fungus interactions. ${ }^{7}$

\section{History}

P. marneffei has been isolated from the organs of apparently healthy bamboo rats and the soil around their burrows but their role in human infection is unclear. ${ }^{8}$ Human penicilliosis marneffei first came in picture as a laboratory-acquired infection when researcher accidentally got needle stick injury when trying to inoculate hamsters with a needle filled with $P$. marneffei.$^{9,10}$ The majority of infections by P. marneffei were diagnosed in AIDS patients in southeast Asia region that include certain parts of India and Thailand however, similar cases were reported from countries like Cambodia, China, Hong Kong, Malaysia, Taiwan and Vietnam..$^{1,11,12}$ Cases from outside endemic region were found in HIV-infected patients from Australia, France, Germany, Japan, the United Kingdom, and the United States. ${ }^{13}$ However, the places where HIV cases are reducing by adopting various preventive social and medical initiatives there are concomitant decreases in the numbers of cases of penicilliosis marneffei. ${ }^{14}$

\section{Mycology}

Penicillium marneffei is a type of dimorphic fungi. ${ }^{3}$ At a temperature range of $23-25^{\circ} \mathrm{C}$, the fungus shows mycelia growth with septate hyphae, containing conidiophores and conidia similar to that of other Penicillium. At $37^{\circ} \mathrm{C}$ the fungus shows growth as yeast where fission arthroconidium cells can be well appreciated and similar cells can be found in the intracellular infection of the macrophages. The mold-to-yeast conversion is a diagnostic characteristic of $P$. marneffei in contrast to other Penicillium species with no dimorphism. ${ }^{3}$ Both mycelia and yeast of $P$. marneffei produces alkaline phosphatase, acid phosphatase, and naphthol ASBI phosphohydrolase. Active esterases, lipases, and galactosidases are also found in some isolates. These enzymes contribute for the virulence in other microorganisms, for example, acid phosphatase in Coxiella burnetti and phospholipase and esterase in Candida albicans. ${ }^{15}$ On the basis of biochemical properties of P. marneffei have 32 isolates. All isolates assimilated glucose, maltose, and cellobiose with some heterogeneity among them. 


\section{Laboratory diagnosis}

\section{Specimens}

Diagnosis has been made most frequently from smears of skin lesions and biopsy samples of lymph node and bone marrow. The organism has also been noted on peripheral blood smear. Bronchoalveolar lavage, pleural fluid, liver biopsies, cerebrospinal fluid, pharyngeal ulcer scrapings, palatal papule scrapings, urine and stool samples may be used as clinical sample. ${ }^{10}$

\section{Microscopy}

Microscopic examination of clinical materials reveals yeast forms both within phagocytes and extracellularly. The intracellular forms are smaller, resembling Histoplasma capsulatum, whereas the extracellular forms are larger and often have a transverse septum. The extracellular forms may also appear as "sausage forms," consisting of three cells divided by two transverse septa or, rarely, as short hyphae. In patients with fulminant infection, $P$. marneffei could be observed in peripheral blood smears. ${ }^{16}$

\section{Histopathology}

Histopathological sections may be stained with hematoxylin and eosin, Grocott methenamine silver, or periodic acid-Schiff stain. One can appreciate fission arthroconidia or oval cells divide by cross wall formation in macrophages. ${ }^{16,17}$ The cross wall formation may differentiate yeast cells of P. marneffei from those of Histoplasma capsulatum. Extracellular elongated or sausage-shaped cells of $P$. marneffei, with one or two septa, may also be seen..$^{10}$ P. marneffei in paraffin-embedded, formalin fixed tissue could be identified by the use of a monoclonal antibody directed to Aspergillus galactomannan. ${ }^{18}$ Here, it seems that there is at least one identical epitope in P. marneffei and Aspergillus spp. but these two fungi have different morphologies as seen in tissue sections. On skin biopsies intracellular yeasts could be clearly demonstrated. Three types of histopathologic reactions can be made in association with $P$. marneffei infection: granulomatous, suppurative, and necrotizing inflammation. Granulomatous or suppurative changes are most commonly seen in patients with normal immunity. The necrotizing reaction is more commonly seen in immunocompromised patients and is characterized by focal necrosis with surrounding histiocytes and extracellular fungi. ${ }^{19}$ Indirect fluorescent-antibody technique may be performed for the rapid identification of $P$. marneffei in histologic sections by using rabbit antiglobulins against yeast culture filtrate antigens of $P$. marneffei.

\section{Culture \& serologic diagnosis}

The fungus grows as mycelial at $25^{\circ} \mathrm{C}$ on Sabouraud Dextrose Agar without cycloheximide. Mold-to-yeast conversion is achieved by subculturing onto brain heart infusion agar and incubating at $37^{\circ} \mathrm{C}$ Identification of P. marneffei is based upon the morphology of the colony, its mold-to-yeast conversion, and the organism's microscopic morphology. A specific exo-antigen test using the immunodiffusion (ID) technique can also be used to identify $P$. marneffei. The concentrated culture filtrate from 6-week-old, standard strain of $P$. marneffei was used as antigen. Rabbit antiglobulin raised against this antigen was used in an ID test to identify P. marneffei. Specific precipitin lines are seen in a positive reaction and the exoantigen test can be used to differentiate $P$. marneffei from other Penicillium species.
An indirect fluorescent-antibody test for detecting IgG antibodies in patients infected with $P$. marneffei may be performed by using germinating conidia and yeast forms as antigens. Immunoblot assay may also be performed by using same lot of antigens. ${ }^{19}$ An enzyme-linked immunosorbent assay (ELISA)-based antibody test was developed and can be used with a recombinant $P$. marneffei mannoprotein (Mplp) for the serodiagnosis of P. marneffei infection. Other test such as specific latex agglutination (LA) for the detection of $P$. marneffei antigens can also be performed. ${ }^{20}$ All tests were highly sensitive and specific.

\section{Molecular diagnosis}

To detect $P$. marneffei, specific oligonucleotide primers designed from the internally transcribed spacer and 5.8S rRNA gene of $P$. marneffei are used ${ }^{20}$ to perform nested PCR; fungal DNA are first amplified with the primer pair ITS5 and ITS4 and second cycle of amplification subsequently performed with primer pair PM1 and PM4 or PM2 and PM4.

\section{Acknowledgements}

None.

\section{Conflict of interest}

The author declares no conflict of interest.

\section{References}

1. Segretain G. Description d'une nouvelle esp'ece de penicillium: Penicillium marneffei n. sp. Bull Soc Mycol Fr. 1959;75:412-416.

2. Sandle T. Dimorph and Filamentous Fungi. In: Mascellino MT, editor. Bacterial and mycotic infections in immunocompromised hosts: clinical and microbiological aspects. USA: OMICS Group Inc; 2013.

3. Chander J. Textbook of Medical Mycology. 3rd ed. New Delhi: Mehta Publishers; 2008.

4. Ranjana KH, Priyokumar K, Singh TJ, et al. Disseminated Penicillium marneffei infection among HIV-infected patients in Manipur state, India. $J$ Infect. 2002;45(4):268-271.

5. Sobottka I, Albrecht H, Mack D, et al. Systemic Penicillium marneffei infection in a German AIDS patient. Eur J Clin Microbiol Infect Dis. 1996;15(3):256-259.

6. Gugnani H, Fisher MC, Paliwal Johsi A, et al. Cannomys badius as a natural animal host of Penicillium marneffei in India. $J$ Clin Microbiol. 2004;42(11):5070-5075.

7. Fisher MC, Aanensen D, De Hoog S, et al. Multilocus microsatellite typing system for Penicillium marneffei reveals spatially structured populations. $J$ Clin Microbiol. 2004;42(11):5065-5069.

8. Capponi M, Sureau P, Segretain G. Penicillose de Rhizomys sinensis. Bull Soc Pathol Exot. 1956;49:418-421.

9. Segretain G. Penicillium marneffei n. sp., agent d'une mycose du systeme reticuloendothelial. Mycopathol Mycol Appl. 1959;11:327-353.

10. Drouhet E. Penicilliosis due to Penicillium marneffei:a new emerging systemic mycosis in AIDS patients travelling or living in Southeast Asia. Review of 44 cases reported in HIV infected patients during the last 5 years compared to 44 cases of non AIDS patients reported over 20years. J Mycol Med (Paris). 1993;4:195-224.

11. Heath TC, Patel A, Fisher D, et al. Disseminated Penicillium marneffei presenting illness of advanced HIV infection: a clinicopathological review, illustrated by a case report. Pathology. 1995;27(1):101-105. 
12. Mohri S, Yoshikawa K, Sagara H, et al. A case of Penicillium marneffei infection in an AIDS patient: the first case in Japan. Nippon Ishinkin Gakkai Zasshi. 2000;41(1):23-26.

13. Huynh TX, Nguyen HC, Dinh Nguyen HM, et al. Penicillium marneffei infection and AIDS. a review of 12 cases reported in the tropical diseases centre, Ho Chi Minh City (Vietnam). Sante. 2003;13(3):149-153.

14. Nord J, Karter D, LaBombardi V. An AIDS patient with fever and pancytopenia. Int J Infect Dis. 1998;2(3):173-175.

15. Tsuboi R, Komatsuzaki H, Ogawa H. Induction of an extra cellular esterase from Candida albicans and some of its properties. Infect Immun. 1996;64(8):2936-2940.

16. Ukarapol N, Sirisanthana V, Wongsawasdi L. Penicillium marneffei mesenteric lymphadenitis in human immunodeficiency virus-infected children. J Med Assoc Thai. 1998;81(8):637-640.
17. Supparatpinyo K, Chiewchanvit S, Hirunsri P, et al. Penicillium marneffei infection in patients infected with human immunodeficiency virus. Clin Infect Dis. 1992;14(4):871-874.

18. Ukarapol N, Sirisanthana V, Wongsawasdi L. Penicillium marneffei mesenteric lymphadenitis in human immunodeficiency virus-infected children. J Med Assoc Thai. 1998;81(8):637-640.

19. Estrada JA, Stynen D, Van Cutsem J, et al. Immunohistochemical identification of Penicillium marneffei by monoclonal antibody. Int $J$ Dermatol. 1992;31(6):410-412.

20. Yuen KY, Wong SS, Tsang DN, et al. Serodiagnosis of Penicillium marneffei infection. Lancet. 1994;344(8920):444-445. 